\title{
POLYDISPERSE HARD-SPHERE YUKAWA CHAIN FLUID: HIGH TEMPERATURE APPROXIMATION
}

\author{
S. P. Hlushak, Yu. V. Kalyuzhnyi \\ Institute for Condensed Matter Physics, \\ 1 Svientsitskoho St., 79011 Lviv, Ukraine
}

(Received January 10, 2007)

\begin{abstract}
The high temperature approximation (HTA) for a polydisperse hard-sphere Yukawa chain fluid is proposed. A closed form analytical expression for Laplace transform of the reference system sitesite radial distribution function, which is used as an input in the theoretical scheme developed, is obtained from the solution of the averaged version of the multidensity Ornstein-Zernike equation. It is demonstrated that in the frames of the HTA polydisperse hard-sphere Yukawa chain fluid model belongs to a class of "truncatable free energy models", i. e. the models with thermodynamical properties (Helmholtz free energy, chemical potential and pressure) defined by a finite number of generalized moments. The accuracy of the theory is accessed by comparison of its liquid-gas phase diagram predictions obtained in the monodisperse limiting case, with the corresponding predictions of the statistical associating fluid theory for the potentials of variable range (SAFTVR) and thermodynamic perturbation theory dimer (TPTD).

Key words: hard-sphere, Yukawa chain fluid, high temperature approximation, Laplace trans-
\end{abstract} form, radial distribution function, Ornstein-Zernike equation.

PACS number(s): 61.20.Gy, 61.25.Em, 61.25.Hq, 83.80.Rs

\section{INTRODUCTION}

It is our pleasure to dedicate this paper to our mentor and colleague Professor Ivan Vakarchuk on the occasion of his 60th birthday.

A vast majority of colloidal and polymeric systems are intrinsically polydisperse, i. e. each particle of a colloidal suspension or polymer blend is unique in its size, charge, shape and other properties. This feature affects the structure and thermodynamic properties of the systems, in particular their phase behavior. Usually the theoretical concepts used to study polydisperse systems treat such systems as a mixture with an infinite number of components, each of them characterized by the variable $x$, which is distributed according to the distribution function $f(x)$. The main obstacle in theoretical description of the phase behavior of polydisperse mixtures is the fact that we are now dealing with a formally infinite number of coexisting equations for the coexisting phases. In spite of the fact that a basic formalism for polydisperse systems was developed some time ago [1], a numerical solution of this problem for a general system, where pressure and chemical potential have to be calculated numerically (with some suitable liquid state theory) remains up to date unsolved. Presently systematic study of the phase behavior of polydisperse fluids is conceivable in the frames of the so-called truncatable free energy models. These are the models with thermodynamic and structure properties expressed via a finite number of generalized moments of the distribution function $f(x)$. As a result infinitely many coexistence equations for the phase equilibrium can be mapped onto a finite number of coupled, highly non-linear equations for these moments. Phase be- havior of a number of different polydisperse systems has been studied via this access [2-5]. However, most of these studies in their sophistication do not go beyond a simple van der Waals (vdW) approach. Only recently in an attempt to go beyond the vdW picture, phase behavior of polydisperse mixtures of hard-sphere Yukawa $[6,7]$ and charged hard-sphere fluids [8-10] have been studied using the high temperature approximation (HTA) and mean spherical approximation (MSA).

In this paper we propose an extension of the HTA for a polydisperse mixture of Yukawa hard-sphere chain molecules. We have shown that within this extension the model at hand belongs to the family of TFE models. Currently there are several theoretical methods which are capable of giving reasonably accurate predictions for thermodynamical properties of the fluid of chain molecules. These include a generalized Flory-dimer theory [11], polymer reference interaction site model (PRISM) equation approach [12], statistical associating fluid theory for the potentials of variable range (SAFT-VR) approach $[13,14]$ and product reactant Ornstein-Zernike approach (PROZA) [15-19]. Among those perhaps only appropriately reformulated SAFT-VR approach can be used to express thermodynamic properties of polydisperse Yukawa hard-sphere chain fluid in terms of finite number of the moments of a certain distribution function. Both SAFT-VR approach and our version of the HTA account interparticle correlations on the level of the corresponding reference system. In the former case the reference system is represented by the Yukawa hardsphere mixture and in the latter case by the hard-sphere chain mixture. We are expecting that HTA predictions will be more accurate of the two, since in the HTA better 


\section{S. P. HLUSHAK, Yu. V. KALYUZHNYI}

approximation for the structure of the original Yukawa hard-sphere chain fluid is used. Laplace transforms of the reference system site-site radial distribution functions (RDF), which are needed in HTA as an input, were calculated analytically using PROZA $[15,16]$ appropriately reformulated in terms of the average total and direct correlation functions. For thermodynamical properties of the reference system we have used thermodynamic perturbation theory (TPT) of Wertheim [20,21], generalized for polydisperse hard-sphere chain mixture. Finally, to verify the accuracy of the present theory we compare our results for the phase diagram of monodisperse Yukawa hard-sphere chain fluid with the corresponding results of other theories.

\section{THE MODEL AND THEORY}

We consider $N$-component mixture of freely jointed tangent hard-sphere Yukawa chain molecules of the species number density $\rho_{a}$ and chain length $m_{a}$. Hereafter small characters $a, b, c, d, \ldots$ denote the species of the chain molecules and take the values $1 \ldots N$, and small characters $i, j, k, \ldots$ indicate the chain monomer species and take the values $1, \ldots, m_{a}$. All the monomers in the chain of the $a$ sort are assumed to be of the same size $\sigma_{a}$. In addition to the hard-sphere interaction there is a Yukawa potential acting between the monomers, which are not connected directly. Thus for the total hard-sphere site-site Yukawa potential we have

$$
u_{Y}^{a b}(r)= \begin{cases}\infty, & r \leq \sigma_{a b} \\ -\frac{\epsilon_{0}^{a b} r_{0}}{r} e^{-z_{0}\left(r-\sigma_{a b}\right)}, & r>\sigma_{a b}\end{cases}
$$

where $\epsilon_{0}$ represents the energy at the contact, $z_{0}$ is the Yukawa screening length and $r_{0}$ is the distance unit. For simplicity reasons we employed here only one-Yukawa interaction; extension of the theory to include multiYukawa potential is rather straightforward. For further simplification we also assume that Yukawa interaction (1) depends only on the species of the chains.

According to the HTA Helmholtz free energy per unit volume can be written as follows

$$
f=f_{\text {ref }}+\Delta f_{\mathrm{HTA}}
$$

where $f_{\text {ref }}$ is Helmholtz free energy density of the reference system, which is represented by the fluid of hardsphere chains, and $\Delta f$ is a contribution from the Yukawa interaction averaged over the reference system RDF:

$$
\Delta f_{\mathrm{HTA}}=2 \pi \sum_{a} \sum_{b} \rho_{a} m_{a} \rho_{b} m_{b} \int_{0}^{\infty} d r r^{2} u_{Y}^{a b}(r) g_{\mathrm{ref}}^{a b}(r) .
$$

where

$$
g_{\mathrm{ref}}^{a b}(r)=\frac{1}{m_{a} m_{b}} \sum_{a b} g_{i j}^{a b}(r)
$$

and $g_{i j}^{a b}(r)$ is site-site RDF between the monomers of $i$ and $j$ species, which belong to the chains of species $a$ and $b$.

Substituting (1) into (3) we have

$$
\Delta f_{\mathrm{HTA}}=-2 \pi \sum_{a} \sum_{b} \rho_{a} m_{a} \rho_{b} m_{b} \epsilon_{0}^{a b} r_{0} e^{z_{0} \sigma_{a b}} G_{\mathrm{ref}}^{a b}\left(z_{0}\right),
$$

where

$$
G_{\mathrm{ref}}^{a b}(s)=\int_{0}^{\infty} d r r e^{-s r} g_{\mathrm{ref}}^{a b}(r)
$$

is the Laplace transform of the RDF $g_{\text {ref }}^{a b}(r)$.

Thermodynamical properties of the reference system will be calculated using TPT of Wertheim [20,21]

\section{STRUCTURE PROPERTIES OF THE REFERENCE SYSTEM}

We will calculate Laplace transform of the RDF $g_{\mathrm{ref}}^{a b}(r)$ using the averaged version of the multidensity OZ equation supplemented by the ideal chain approximation and polymer Percus-Yevick (PPY) closure relation $[15,16]$.

\section{A. Averaged multidensity $\mathrm{OZ}$ equation in the ideal chain approximation}

Multidensity OZ equation for the multicomponent chain fluid in the ideal chain approximation is $[15,16]$ :

$$
\mathbf{h}_{i j}^{a b}(r)=\mathbf{c}_{i j}^{a b}(r)+\sum_{c, k} \int\left[\mathbf{c}_{i k}^{a c}\left(r^{\prime}\right)+\Delta_{i k}^{a c}\left(r^{\prime}\right)\right] \rho_{c} \alpha\left[\mathbf{h}_{k j}^{c b}\left(\left|\mathbf{r}-\mathbf{r}^{\prime}\right|\right)+\boldsymbol{\Delta}_{k j}^{c b}\left(\left|\mathbf{r}-\mathbf{r}^{\prime}\right|\right)\right] d \mathbf{r}^{\prime}
$$

where we have extracted from the correlation functions the terms with delta functions and denoted them by $\boldsymbol{\Delta}_{i j}^{a b}(r)$. The matrices used in (6) are total correlation function matrix

$$
\mathbf{h}_{i j}^{a b}(r)=\left(\begin{array}{ccc}
h_{i j, 00}^{a b}(r) & h_{i j, 0 A}^{a b}(r) & h_{i j, 0 B}^{a b}(r) \\
h_{i j, A 0}^{a b}(r) & h_{i j, A A}^{a b}(r) & h_{i j, A B}^{a b}(r) \\
h_{i j, B 0}^{a b}(r) & h_{i j, B A}^{a b}(r) & h_{i j, B B}^{a b}(r)
\end{array}\right),
$$

direct correlation function matrix

$$
\mathbf{c}_{i j}^{a b}(r)=\left(\begin{array}{ccc}
c_{i j, 00}^{a b}(r) & c_{i j, 0 A}^{a b}(r) & c_{i j, 0 B}^{a b}(r) \\
c_{i j, A 0}^{a b}(r) & c_{i j, A A}^{a b}(r) & c_{i j, A B}^{a b}(r) \\
c_{i j, B 0}^{a b}(r) & c_{i j, B A}^{a b}(r) & c_{i j, B B}^{a b}(r)
\end{array}\right),
$$




$$
\alpha=\left(\begin{array}{lll}
1 & 1 & 1 \\
1 & 0 & 1 \\
1 & 1 & 0
\end{array}\right)
$$

and the matrix containing delta-functions:

$$
\boldsymbol{\Delta}_{i j}^{a b}(r)=\delta_{i, j+1}\left(\begin{array}{ccc}
0 & 0 & 0 \\
0 & 0 & \Delta^{a b}(r) \\
0 & 0 & 0
\end{array}\right)
$$

$$
+\delta_{i, j-1}\left(\begin{array}{ccc}
0 & 0 & 0 \\
0 & 0 & 0 \\
0 & \Delta^{a b}(r) & 0
\end{array}\right)
$$

where

$$
\Delta^{a b}(r)=\frac{\delta_{a b}}{4 \pi \rho_{a} \sigma_{a b}^{2}} \delta\left(r-\sigma_{a b}\right), \quad \sigma_{a b}=\left(\sigma_{a}+\sigma_{b}\right) / 2
$$

Taking the average of both sides of equation (6) we have

$$
\begin{aligned}
\frac{1}{m_{a} m_{b}} \sum_{i j} \mathbf{h}_{i j}^{a b}(r) & =\frac{1}{m_{a} m_{b}} \sum_{i j} \mathbf{c}_{i j}^{a b}(r) \\
& +\frac{1}{m_{a} m_{b}} \sum_{i j} \sum_{c k} \int\left[\mathbf{c}_{i k}^{a c}\left(r^{\prime}\right)+\boldsymbol{\Delta}_{i k}^{a c}\left(r^{\prime}\right)\right] \rho_{c} \alpha\left[\mathbf{h}_{k j}^{c b}\left(\left|\mathbf{r}-\mathbf{r}^{\prime}\right|\right)+\mathbf{\Delta}_{k j}^{c b}\left(\left|\mathbf{r}-\mathbf{r}^{\prime}\right|\right)\right] d \mathbf{r}^{\prime}
\end{aligned}
$$

To proceed we will assume that site-site correlation functions $\mathbf{c}_{i j}^{a b}(r)$ and $\mathbf{h}_{i j}^{a b}(r)$ are independent of the positions of the sites $(a, i)$ and $(b, j)$ in the chains $a$ and $b$. By direct multiplication and summation it can be shown that

$$
\frac{1}{m_{a} m_{b}} \sum_{i j} \sum_{c k} \boldsymbol{\Delta}_{i k}^{a c}\left(r^{\prime}\right) \rho_{c} \alpha \boldsymbol{\Delta}_{k j}^{c b}\left(\left|\mathbf{r}-\mathbf{r}^{\prime}\right|\right)=\sum_{c} \frac{m_{c}^{2}\left(m_{c}-2\right)}{\left(m_{c}-1\right)^{2}} \bar{\Delta}^{a c}\left(r^{\prime}\right) \rho_{c} \alpha \bar{\Delta}^{c b}\left(\left|\mathbf{r}-\mathbf{r}^{\prime}\right|\right)
$$

where we have introduced a new matrix

$$
\bar{\Delta}^{a b}(r)=\left(\begin{array}{ccc}
0 & 0 & 0 \\
0 & 0 & \bar{\Delta}^{a b}(r) \\
0 & \bar{\Delta}^{a b}(r) & 0
\end{array}\right)
$$

with

$$
\bar{\Delta}^{a b}(r)=\frac{\left(m_{a}-1\right) \delta_{a b}}{4 \pi m_{a}^{2} \rho_{a} \sigma_{a b}^{2}} \delta\left(r-\sigma_{a b}\right)
$$

Similarly

$$
\begin{aligned}
& \frac{1}{m_{a} m_{b}} \sum_{i j} \sum_{c k} \boldsymbol{\Delta}_{i k}^{a c}\left(r^{\prime}\right) \rho_{c} \alpha \mathbf{h}_{k j}^{c b}\left(\left|\mathbf{r}-\mathbf{r}^{\prime}\right|\right)=\sum_{c} \overline{\boldsymbol{\Delta}}^{a c}\left(r^{\prime}\right) \rho_{c} \alpha \overline{\mathbf{h}}^{c b}\left(\left|\mathbf{r}-\mathbf{r}^{\prime}\right|\right), \\
& \frac{1}{m_{a} m_{b}} \sum_{i j} \sum_{c k} \mathbf{c}_{i k}^{a c}\left(r^{\prime}\right) \rho_{c} \alpha \boldsymbol{\Delta}_{k j}^{c b}\left(\left|\mathbf{r}-\mathbf{r}^{\prime}\right|\right)=\sum_{c} \overline{\mathbf{c}}^{a c}\left(r^{\prime}\right) \rho_{c} \alpha \overline{\boldsymbol{\Delta}}^{c b}\left(\left|\mathbf{r}-\mathbf{r}^{\prime}\right|\right),
\end{aligned}
$$

where $\overline{\mathbf{c}}^{c b}(r)$ and $\overline{\mathbf{h}}^{c b}(r)$ are the averaged direct and total correlation functions

$$
\begin{aligned}
\overline{\mathbf{c}}^{a b}(r) & =\frac{1}{m_{a} m_{b}} \sum_{i j} \mathbf{c}_{i k}^{a b}(r), \\
\overline{\mathbf{h}}^{a b}(r) & =\frac{1}{m_{a} m_{b}} \sum_{i j} \mathbf{h}_{i k}^{a b}(r) .
\end{aligned}
$$

Note that $\bar{\Delta}^{a b}(r)$ has a Kroneker delta symbol $\delta_{a b}$, thus the sums over $c$ in (16) and (17) can be removed. However, we keep them because they will be used to assemble the OZ equation from the corresponding averaged quantities.

$$
\begin{aligned}
\overline{\mathbf{h}}^{a b}(r)= & \overline{\mathbf{c}}^{a b}(r)+\sum_{c} \rho_{c} m_{c} \int\left[\overline{\mathbf{c}}^{a c}\left(r^{\prime}\right) \alpha \overline{\mathbf{h}}^{c b}\left(\left|\mathbf{r}-\mathbf{r}^{\prime}\right|\right)+\overline{\mathbf{c}}^{a c}\left(r^{\prime}\right) \alpha \overline{\boldsymbol{\Delta}}^{c b}\left(\left|\mathbf{r}-\mathbf{r}^{\prime}\right|\right)\right. \\
& \left.+\overline{\boldsymbol{\Delta}}_{i k}^{a c}\left(r^{\prime}\right) \alpha \overline{\mathbf{h}}_{k j}^{c b}\left(\left|\mathbf{r}-\mathbf{r}^{\prime}\right|\right)+\frac{m_{c}\left(m_{c}-2\right)}{\left(m_{c}-1\right)^{2}} \overline{\boldsymbol{\Delta}}_{i k}^{a c}\left(r^{\prime}\right) \alpha \overline{\boldsymbol{\Delta}}_{k j}^{c b}\left(\left|\mathbf{r}-\mathbf{r}^{\prime}\right|\right)\right] d \mathbf{r}^{\prime}
\end{aligned}
$$




\section{S. P. HLUSHAK, Yu. V. KALYUZHNYI}

Neglecting in (20) the terms with $1 / m_{c}^{2}$ we have

$$
\frac{m_{c}\left(m_{c}-2\right)}{\left(m_{c}-1\right)^{2}}=1
$$

and finally equation (20) can be written in the following form

$$
\mathbf{h}^{a b}(r)=\mathbf{c}^{a b}(r)+\sum_{c} \rho_{c}^{(m)} \int \mathbf{c}^{a c}\left(r^{\prime}\right) \alpha \mathbf{h}^{c b}\left(\left|\mathbf{r}-\mathbf{r}^{\prime}\right|\right) d \mathbf{r}^{\prime},
$$

where $\rho_{c}^{(m)}$ is the number density of the monomers, which belong to the chain of the $c$ sort, i. e. $\rho_{c}^{(m)}=m_{c} \rho_{c}$, and

$$
\begin{aligned}
\mathbf{c}^{a b}(r) & =\overline{\mathbf{c}}^{a c}(r)+\overline{\boldsymbol{\Delta}}^{a c}(r), \\
\mathbf{h}^{a b}(r) & =\overline{\mathbf{h}}^{c b}(r)+\overline{\boldsymbol{\Delta}}^{c b}(r),
\end{aligned}
$$

are the new averaged correlation functions, which now depend only on the chain species indices. For the corresponding version of the PPY approximation we have

$$
\begin{aligned}
h_{\alpha \beta}^{a b}(r) & =-\delta_{\alpha 0} \delta_{\beta 0}, \quad r<\sigma_{a b}, \\
c_{\alpha \beta}^{a b}(r) & =\frac{\delta_{a b}\left(m_{a}-1\right)}{4 \pi \rho_{a}^{(m)} m_{a}\left(\sigma_{a b}\right)^{2}}\left(\delta_{\alpha A} \delta_{\beta B}+\delta_{\alpha B} \delta_{\beta A}\right) \delta\left(r-\sigma_{a b}\right), \quad r \geq \sigma_{a b}
\end{aligned}
$$

where $\alpha$ and $\beta$ take the values $0, A, B$.

We note in passing that the averaged OZ equation (22) coincides with multidensity OZ equation for the fluid of associating particles forming chains with the average length $m_{a}[22,23]$.

\section{B. Solution of the averaged $\mathrm{OZ}$ equation}

Solution of the set of the OZ equation (22) with closure conditions (25) and (26) was performed employing Baxter factorization method [24], earlier [22,23]. Therefore, we will omit details here and present only the final result, which will be needed to derive an expression for Laplace transform of the RDF. Factorizing set of equations (22) we have

$$
\begin{gathered}
-r c_{\alpha \beta}^{a b}(r)=\left[q_{\alpha \beta}^{a b}(r)\right]^{\prime}-2 \pi \sum_{c} \rho_{c} m_{c} \sum_{\gamma, \delta}\left(1-\delta_{\gamma \delta}+\delta_{\gamma 0} \delta_{\delta 0}\right) \frac{\partial}{\partial r} \int_{\lambda_{a c}}^{\min \left[\sigma_{\mathrm{ac}}, \sigma_{\mathrm{cb}}-\mathrm{r}\right]} q_{\gamma \alpha}^{c a}(t) q_{\delta \beta}^{c b}(r+t) d t, \\
\text { for } \quad \lambda_{a b}<r<\sigma_{a b}, \\
-r h_{\alpha \beta}^{a b}(r)=\left[q_{\alpha \beta}^{a b}(r)\right]^{\prime}-2 \pi \sum_{c} \rho_{c} m_{c} \sum_{\gamma, \delta}\left(1-\delta_{\gamma \delta}+\delta_{\gamma 0} \delta_{\delta 0}\right) \int_{\lambda_{a c}}^{\sigma_{a c}} q_{\alpha \gamma}^{a c}(t)(r-t) h_{\delta \beta}^{c b}(|r-t|) d t, \\
r>\lambda_{a b},
\end{gathered}
$$

where $\lambda_{a b}=\frac{1}{2}\left(\sigma_{a}-\sigma_{b}\right)$ and

$$
q_{\alpha \beta}^{a b}(r)=\left(\frac{1}{2} a_{\alpha}^{(a)} r^{2}+b_{\alpha}^{(a)} r\right) \delta_{\beta 0}+d_{\alpha \beta}^{a b}
$$

This function is defined in the interval $\lambda_{a b}<r<\sigma_{a b}$, with the coefficients $a_{\alpha}^{(a)}, b_{\alpha}^{(a)}$ and $d_{\alpha \beta}^{a b}$ found to be

$$
\begin{aligned}
& a_{0}^{(a)}=\frac{1}{\Delta}+\frac{1}{2} \frac{\sigma_{a} \xi_{2}}{\Delta^{2}}, \quad b_{0}^{(a)}=-\frac{1}{4} \frac{\left(\sigma_{a}\right)^{2} \xi_{2}}{\Delta^{2}}, \quad d_{00}^{a b}=-\frac{1}{2} \frac{\left(\sigma_{a}\right)^{2} \xi_{2}}{\Delta}+\frac{1}{4} \frac{\sigma_{a} \sigma_{a b} \lambda_{a b} \xi_{2}}{\Delta^{2}}, \\
& a_{M}^{(a)}=-\frac{1}{2} \frac{\left(m_{a}-1\right)}{m_{a} \Delta}\left(\delta_{M A}+\delta_{M B}\right), \quad b_{M}^{(a)}=-\frac{1}{2} a_{M}^{(a)} \sigma_{a}, \quad d_{M 0}^{a b}=\frac{1}{2} a_{M}^{(a)} \sigma_{a b} \lambda_{a b} \\
& d_{0 L}^{a b}=0, \quad d_{M L}^{a b}=\frac{\delta_{a b}}{4 \pi \rho_{a} m_{a} \sigma_{a}}\left(\frac{m_{a}-1}{m_{a}}\right)\left(\delta_{M A} \delta_{L B}+\delta_{L A} \delta_{M B}\right)
\end{aligned}
$$

where $\xi_{i}=\pi \sum_{c} \rho_{c} m_{c} \sigma_{c}^{i}, \Delta=1-\xi_{3} / 6$ and $M$ and $L$ take the values $A$ and $B$.

Expressions for the regular part of contact values of the $\mathrm{RDF} g_{\alpha \beta}^{a b}(r)=\delta_{\alpha 0} \delta_{\beta 0}+h_{\alpha \beta}^{a b}(r)$ are: 


$$
\begin{aligned}
g_{00}^{a b}\left(\sigma_{a b}+\right) & =\frac{1}{\Delta}+\frac{1}{4} \frac{\sigma_{a} \sigma_{b} \xi_{2}}{\Delta^{2} \sigma_{a b}}, \\
g_{M 0}^{a b}\left(\sigma_{a b}+\right) & =-\frac{1}{4} \frac{\sigma_{b}}{\Delta \sigma_{a b}}\left(\frac{m_{a}-1}{m_{a}}\right), \\
g_{0 M}^{a b}\left(\sigma_{a b}+\right) & =-\frac{1}{4} \frac{\sigma_{a}}{\Delta \sigma_{a b}}\left(\frac{m_{b}-1}{m_{b}}\right), \\
g_{M L}^{a b}\left(\sigma_{a b}+\right) & =\frac{\delta_{a b}}{8 \pi \rho_{a} m_{a} \sigma_{a}^{2} \sigma_{a b}}\left(\frac{m_{a}-1}{m_{a}}\right)^{2}\left(1-\delta_{M L}\right)
\end{aligned}
$$

\section{Laplace transform of the site-site radial distribution functions}

Integrating both sides of equation (28) with $\int_{\lambda_{a b}}^{\infty} e^{-s r} \ldots d r$ we have

$$
\begin{aligned}
& \sum_{c} \sum_{\delta}\left(\delta_{c a} \delta_{\delta \alpha}-2 \pi \rho_{c} m_{c}\left[Q_{\alpha 0}^{a c}(s)+\left(1-\delta_{\delta A}\right) Q_{\alpha A}^{a c}(s)+\left(1-\delta_{\delta B}\right) Q_{\alpha B}^{a c}(s)\right]\right) G_{\delta \beta}^{c b}(s) \\
& =\delta_{\alpha 0} \delta_{\beta 0} \int_{\lambda_{a b}}^{\infty} e^{-s r} d r-Q_{\alpha \beta}^{a b}{ }^{\prime}(s)-2 \pi \sum_{c} \rho_{c} m_{c} \sum_{\gamma \delta}\left(1-\delta_{\gamma \delta}+\delta_{\gamma 0} \delta_{\delta 0}\right) \delta_{\delta 0} \delta_{\beta 0} \\
& \times \int_{\lambda_{a c}}^{\sigma_{a c}} q_{\alpha \gamma}^{a c}(t)\left(\frac{1}{s^{2}}-\frac{\lambda_{a b}}{s}-\frac{t}{s}\right) e^{-s \lambda_{a b}} d t
\end{aligned}
$$

where

$$
\begin{aligned}
& Q_{\alpha \beta}^{a b}(s)=\int_{\lambda_{a b}}^{\infty} e^{-s r} q_{\alpha \beta}^{a b}(r) d r=e^{-s \lambda_{a b}}\left(\left[a_{\alpha}^{(a)}\left(\varphi_{2}\left(\sigma_{b}\right)+\frac{\sigma_{b}}{2} \varphi_{1}\left(\sigma_{b}\right)\right)\right.\right. \\
&\left.\left.+\left(\frac{a_{\alpha}^{(a)} \sigma_{a}}{2}+b_{\alpha}^{(a)}\right) \varphi_{1}\left(\sigma_{b}\right)\right] \delta_{\beta 0}+\left(1-\delta_{\alpha 0}\right)\left(1-\delta_{\beta 0}\right) d_{\alpha \beta}^{a b} \varphi_{0}\left(\sigma_{b}\right)\right) \\
& Q_{\alpha \beta}^{a b{ }^{\prime}}(s)=\int_{\lambda_{a b}}^{\infty} e^{-s r} q_{\alpha \beta}^{a b{ }^{\prime}}(r) d r=e^{-s \lambda_{a b}}\left(\left[a_{\alpha}^{(a)}\left(\varphi_{1}\left(\sigma_{b}\right)+\frac{\sigma_{b}}{2} \varphi_{0}\left(\sigma_{b}\right)\right)\right.\right. \\
&\left.\left.+\left(\frac{a_{\alpha}^{(a)} \sigma_{a}}{2}+b_{\alpha}^{(a)}\right) \varphi_{0}\left(\sigma_{b}\right)\right] \delta_{\beta 0}-\left(1-\delta_{\alpha 0}\right)\left(1-\delta_{\beta 0}\right) d_{\alpha \beta}^{a b}\right), \\
& \varphi_{2}\left(\sigma_{b}\right)=\frac{1-s \sigma_{b}+s^{2} \sigma_{b}{ }^{2} / 2-e^{-s \sigma_{b}}}{s^{3}}, \quad \varphi_{1}\left(\sigma_{b}\right)=\frac{1-s \sigma_{b}-e^{-s \sigma_{b}}}{s^{2}}, \quad \varphi_{0}\left(\sigma_{b}\right)=\frac{1-e^{-s \sigma_{b}}}{s},
\end{aligned}
$$

and

$$
G_{\alpha \beta}^{a b}(s)=\int_{\lambda_{a b}}^{\infty} e^{-s r} g_{\alpha \beta}^{a b}(r) d r .
$$

Equation (34) can be written in the following form:

$$
\sum_{c} \sum_{\delta} M_{\alpha \delta}^{a c} G_{\delta \beta}^{c b}(s)=\hat{a}_{\alpha}^{(a)}\left[\frac{1}{s^{2}}+\frac{\sigma_{b}}{2 s}\right] \delta_{\beta 0}+\hat{c}_{\alpha}^{(a)} \frac{1}{s} \delta_{\beta 0}+\sum_{t} \hat{e}_{\alpha}^{(a)} \frac{\delta_{t b} \delta_{\beta A}}{2 \pi \rho_{b} m_{b}} e^{-s \sigma_{b} / 2}+\sum_{t} \hat{g}_{\alpha}^{(a)} \frac{\delta_{t b} \delta_{\beta B}}{2 \pi \rho_{b} m_{b}} e^{-s \sigma_{b} / 2},
$$

where Jacobi like matrix $M_{\alpha \delta}^{a c}$ is given by

$$
M_{\alpha \delta}^{a c}=\delta_{a c} \delta_{\alpha \delta}-\hat{a}_{\alpha}^{(a)} \hat{b}_{\delta}^{(c)}-\hat{c}_{\alpha}^{(a)} \hat{d}_{\delta}^{(c)}-\sum_{t}\left(\hat{e}_{\alpha}^{(a)(t)} \hat{f}_{\delta}^{(c)(t)}+\hat{g}_{\alpha}^{(a)(t)} \hat{h}_{\delta}^{(c)(t)}\right) .
$$

and

$$
\begin{aligned}
& \hat{a}_{\alpha}^{(a)}=e^{-s \sigma_{a} / 2} a_{\alpha}^{(a)} \\
& \hat{b}_{\delta}^{(c)}=2 \pi \rho_{c} m_{c}\left[\varphi_{2}\left(\sigma_{c}\right)+\frac{\sigma_{c}}{2} \varphi_{1}\left(\sigma_{c}\right)\right] e^{s \sigma_{c} / 2},
\end{aligned}
$$




$$
\begin{aligned}
& \hat{c}_{\alpha}^{(a)}=e^{-s \sigma_{a} / 2}\left[\frac{\sigma_{a}}{2} a_{\alpha}^{(a)}+b_{\alpha}^{(a)}\right]=e^{-s \sigma_{b} / 2} \frac{\delta_{\alpha 0} \sigma_{a}}{2 \Delta}, \\
& \hat{d}_{\delta}^{(c)}=2 \pi \rho_{c} m_{c} \varphi_{1}\left(\sigma_{c}\right) e^{s \sigma_{c} / 2}, \\
& \hat{e}_{\alpha}^{(a)(t)}=e^{-s \sigma_{a} / 2} \frac{m_{a}-1}{2 m_{a} \sigma_{a}} \delta_{a t} \delta_{\alpha B}, \\
& \hat{f}_{\delta}^{(c)(t)}=\varphi_{0}\left(\sigma_{c}\right) e^{s \sigma_{c} / 2} \delta_{c t}\left(1-\delta_{\delta A}\right), \\
& \hat{g}_{\alpha}^{(a)(t)}=e^{-s \sigma_{a} / 2} \frac{m_{a}-1}{2 m_{a} \sigma_{a}} \delta_{a t} \delta_{\alpha A}, \\
& \hat{h}_{\delta}^{(c)(t)}=\varphi_{0}\left(\sigma_{c}\right) e^{s \sigma_{c} / 2} \delta_{c t}\left(1-\delta_{\delta B}\right), \text { for } t=1 \ldots N .
\end{aligned}
$$

The last four vectors $\hat{e}_{\alpha}^{(a)(t)}, \hat{f}_{\delta}^{(c)(t)}, \hat{g}_{\alpha}^{(a)(t)}$ and $\hat{h}_{\delta}^{(c)(t)}$ appear due to decomposition of the Kronecker delta

$$
\delta_{a b}=\sum_{t} \delta_{a t} \delta_{t b}
$$

in $d_{\alpha \beta}^{a b}$. This decomposition makes the matrix $M_{\alpha \delta}^{a c}$ Jacobi-like. Thus, similar to [25-27], the solution of equation (34) can be obtained inverting this matrix. In general it appears to be impossible to revert such a matrix with an arbitrary number of forming vectors $\hat{e}_{\alpha}^{(a)(t)}, \hat{f}_{\delta}^{(c)(t)}, \hat{g}_{\alpha}^{(a)(t)}$ and $\hat{h}_{\delta}^{(c)(t)}$ for $t=1 \ldots N$ without knowing that some of these vectors are orthogonal and others can be composed back to the form $\delta_{a b}$ :

$$
\begin{aligned}
\left(\hat{\mathbf{e}}^{(p)} \hat{\mathbf{f}}^{(q)}\right) & =\sum_{c} \sum_{\gamma} \hat{e}_{\gamma}^{(c)(p)} \hat{f}_{\gamma}^{(c)(q)}=\frac{m_{p}-1}{2 m_{p} \sigma_{p}} \varphi_{0}\left(\sigma_{p}\right) \delta_{p q} \\
\left(\hat{\mathbf{e}}^{(p)} \hat{\mathbf{h}}^{(q)}\right) & =\sum_{c} \sum_{\gamma} \hat{e}_{\gamma}^{(c)(p)} \hat{h}_{\gamma}^{(c)(q)}=0 \\
\left(\hat{\mathbf{g}}^{(p)} \hat{\mathbf{f}}^{(q)}\right) & =\sum_{c} \sum_{\gamma} \hat{g}_{\gamma}^{(c)(p)} \hat{f}_{\gamma}^{(c)(q)}=0 \\
\left(\hat{\mathbf{g}}^{(p)} \hat{\mathbf{h}}^{(q)}\right) & =\sum_{c} \sum_{\gamma} \hat{g}_{\gamma}^{(c)(p)} \hat{h}_{\gamma}^{(c)(q)}=\frac{m_{p}-1}{2 m_{p} \sigma_{p}} \varphi_{0}\left(\sigma_{p}\right) \delta_{p q} .
\end{aligned}
$$

We assume the following form for the inverse matrix:

$$
\begin{aligned}
{\left[M^{-1}\right]_{\gamma \lambda}^{x y} } & =\delta_{x y} \delta_{\gamma \lambda}+\alpha_{\hat{a} \hat{b}} \hat{a}_{\gamma}^{(x)} \hat{b}_{\lambda}^{(y)}+\alpha_{\hat{c} \hat{d}} \hat{c}_{\gamma}^{(x)} \hat{d}_{\lambda}^{(y)}+\alpha_{\hat{c} \hat{b}} \hat{c}_{\gamma}^{(x)} \hat{b}_{\lambda}^{(y)}+\alpha_{\hat{a} \hat{d}} \hat{a}_{\gamma}^{(x)} \hat{d}_{\lambda}^{(y)} \\
& +\sum_{t} \hat{a}_{\gamma}^{(x)}\left(\alpha_{\hat{a} \hat{f}}^{(t)} \hat{f}_{\lambda}^{(y)(t)}+\alpha_{\hat{a} \hat{h}}^{(t)} \hat{h}_{\lambda}^{(y)(t)}\right)+\sum_{t} \hat{c}_{\gamma}^{(x)}\left(\alpha_{\hat{c} \hat{f}}^{(t)} \hat{f}_{\lambda}^{(y)(t)}+\alpha_{\hat{c} \hat{h}}^{(t)} \hat{h}_{\lambda}^{(y)(t)}\right) \\
& +\sum_{t}\left(\alpha_{\hat{e} \hat{b}}^{(t)} \hat{e}_{\gamma}^{(x)(t)}+\alpha_{\hat{g} \hat{b}}^{(t)} \hat{g}_{\gamma}^{(x)(t)}\right) \hat{b}_{\lambda}^{(y)}+\sum_{t}\left(\alpha_{\hat{e} \hat{d}}^{(t)} \hat{e}_{\gamma}^{(x)(t)}+\alpha_{\hat{g} \hat{d}}^{(t)} \hat{g}_{\gamma}^{(x)(t)}\right) \hat{d}_{\lambda}^{(y)} \\
& +\sum_{t_{1}} \sum_{t_{2}} \alpha_{\hat{e} \hat{f}}^{\left(t_{1}\right)\left(t_{2}\right)} \hat{e}_{\gamma}^{(x)\left(t_{1}\right)} \hat{f}_{\lambda}^{(y)\left(t_{2}\right)}+\sum_{t_{1}} \sum_{t_{2}} \alpha_{\hat{g} \hat{h}}^{\left(t_{1}\right)\left(t_{2}\right)} \hat{g}_{\gamma}^{(x)\left(t_{1}\right)} \hat{h}_{\lambda}^{(y)\left(t_{2}\right)} \\
& +\sum_{t_{1}} \sum_{t_{2}} \alpha_{\hat{e} \hat{h}}^{\left(t_{1}\right)\left(t_{2}\right)} \hat{e}_{\gamma}^{(x)\left(t_{1}\right)} \hat{h}_{\lambda}^{(y)\left(t_{2}\right)}+\sum_{t_{1}} \sum_{t_{2}} \alpha_{\hat{g} \hat{f}}^{\left(t_{1}\right)\left(t_{2}\right)} \hat{g}_{\gamma}^{(x)\left(t_{1}\right)} \hat{f}_{\lambda}^{(y)\left(t_{2}\right)}
\end{aligned}
$$

where $\alpha_{\hat{a} \hat{b}}, \alpha_{\hat{c} \hat{d}}, \ldots, \alpha_{\hat{g} \hat{f}}^{\left(t_{1}\right)\left(t_{2}\right)}$ are unknown coefficients which follow from the relation $\mathbf{M M}^{-\mathbf{1}}=\mathbf{I}$ with $\mathbf{I}$ being unity matrix. Obtained coefficients are presented in Appendix A.

Multiplying both sides of equation (38) by $\mathbf{M}^{-\mathbf{1}}$ we have:

$$
\begin{aligned}
G_{\alpha \beta}^{a b}(s) & =\frac{e^{-s \sigma_{a b}}}{\Delta D} \delta_{\alpha 0} \delta_{\beta 0}\left[\frac{1}{s^{2}}+\frac{\sigma_{a b}}{s}-\sigma_{a} \sigma_{b} \sum_{c} \frac{\pi \rho_{c} m_{c}}{\Delta s^{2}} \frac{K_{c}}{1-K_{c}} \sigma_{c} \frac{\varphi_{1}\left(\sigma_{c}\right)}{\varphi_{0}\left(\sigma_{c}\right)} s+\frac{\sigma_{a} \sigma_{b} \xi_{2}}{4 \Delta s}\right. \\
& \left.+\frac{1}{2 \Delta s^{2}} \sum_{c} \pi \rho_{c} m_{c} \sigma_{c}^{3} \psi\left(\sigma_{c}\right)+\sum_{c} \frac{\pi \rho_{c} m_{c} \varphi_{1}\left(\sigma_{c}\right) \psi\left(\sigma_{c}\right)}{2 \Delta s}\left(\sigma_{a}-\sigma_{c}\right)\left(\sigma_{b}-\sigma_{c}\right)\right] \\
& +\frac{e^{-s \sigma_{a b}}}{\Delta D} \frac{K_{a}}{1-K_{a}}\left(1-\delta_{\alpha 0}\right) \delta_{\beta 0}\left[\frac{1}{s^{2}}+\frac{\sigma_{a b}}{s}-\frac{\sigma_{a}}{\varphi_{0}\left(\sigma_{a}\right)}\left(\frac{1}{s^{2}}+\frac{\sigma_{b}}{2 s}\right)\right. \\
& +\frac{\sigma_{a}}{2 \Delta s^{2}}\left(\xi_{2}-\sum_{c} \pi \rho_{c} m_{c} \sigma_{c}^{2} \Theta\left(\sigma_{c}\right)\right)+\frac{\sigma_{a} \sigma_{b} \xi_{2}}{4 \Delta s}+\frac{1}{2 \Delta s^{2}} \frac{\varphi_{1}\left(\sigma_{a}\right)}{\varphi_{0}\left(\sigma_{a}\right)} s \sum_{c} \pi \rho_{c} m_{c} \sigma_{c}^{3} \psi\left(\sigma_{c}\right)
\end{aligned}
$$




$$
\begin{aligned}
& \left.+\sum_{c} \frac{\pi \rho_{c} m_{c} \varphi_{1}\left(\sigma_{c}\right)}{2 \Delta s}\left(\sigma_{a} \Theta\left(\sigma_{c}\right)-\frac{\varphi_{1}\left(\sigma_{a}\right)}{\varphi_{0}\left(\sigma_{a}\right)} s \psi\left(\sigma_{c}\right) \sigma_{c}\right)\left(\sigma_{b}-\sigma_{c}\right)\right] \\
& +\frac{e^{-s \sigma_{a b}}}{\Delta D} \frac{K_{b}}{1-K_{b}} \delta_{\alpha 0}\left(1-\delta_{\beta 0}\right)\left[\frac{1}{s^{2}}+\frac{\sigma_{a b}}{s}-\frac{\sigma_{b}}{\varphi_{0}\left(\sigma_{b}\right)}\left(\frac{1}{s^{2}}+\frac{\sigma_{a}}{2 s}\right)\right. \\
& +\frac{\sigma_{b}}{2 \Delta s^{2}}\left(\xi_{2}-\sum_{c} \pi \rho_{c} m_{c} \sigma_{c}^{2} \Theta\left(\sigma_{c}\right)\right)+\frac{\sigma_{a} \sigma_{b} \xi_{2}}{4 \Delta s}+\frac{1}{2 \Delta s^{2}} \frac{\varphi_{1}\left(\sigma_{b}\right)}{\varphi_{0}\left(\sigma_{b}\right)} s \sum_{c} \pi \rho_{c} m_{c} \sigma_{c}^{3} \psi\left(\sigma_{c}\right) \\
& \left.+\sum_{c} \frac{\pi \rho_{c} m_{c} \varphi_{1}\left(\sigma_{c}\right)}{2 \Delta s}\left(\sigma_{b} \Theta\left(\sigma_{c}\right)-\frac{\varphi_{1}\left(\sigma_{b}\right)}{\varphi_{0}\left(\sigma_{b}\right)} s \psi\left(\sigma_{c}\right) \sigma_{c}\right)\left(\sigma_{a}-\sigma_{c}\right)\right] \\
& +\frac{e^{-s \sigma_{a b}}}{\Delta D} \frac{K_{a}}{1-K_{a}} \frac{K_{b}}{1-K_{b}}\left(1-\delta_{\alpha 0}\right)\left(1-\delta_{\beta 0}\right)\left[\frac{\varphi_{1}\left(\sigma_{a}\right)}{\varphi_{0}\left(\sigma_{a}\right)} \frac{\varphi_{1}\left(\sigma_{b}\right)}{\varphi_{0}\left(\sigma_{b}\right)}+\frac{\sigma_{a} \sigma_{b} \xi_{2}}{4 \Delta s}\right. \\
& +\left(\frac{\sigma_{b}}{2} \frac{\varphi_{1}\left(\sigma_{a}\right)}{\varphi_{0}\left(\sigma_{a}\right)}+\frac{\sigma_{a}}{2} \frac{\varphi_{1}\left(\sigma_{b}\right)}{\varphi_{0}\left(\sigma_{b}\right)}\right)+\frac{1}{2 \Delta} \frac{\varphi_{1}\left(\sigma_{a}\right)}{\varphi_{0}\left(\sigma_{a}\right)} \frac{\varphi_{1}\left(\sigma_{b}\right)}{\varphi_{0}\left(\sigma_{b}\right)} \sum_{c} \pi \rho_{c} m_{c} \sigma_{c}^{3} \psi\left(\sigma_{c}\right) \\
& +\frac{s}{2 \Delta} \frac{\varphi_{1}\left(\sigma_{a}\right)}{\varphi_{0}\left(\sigma_{a}\right)} \frac{\varphi_{1}\left(\sigma_{b}\right)}{\varphi_{0}\left(\sigma_{b}\right)} \sum_{c} \pi \rho_{c} m_{c} \varphi_{1}\left(\sigma_{c}\right) \sigma_{c}^{2} \psi\left(\sigma_{c}\right)+\frac{\sigma_{a} \sigma_{b}}{2 \Delta s} \sum_{c} \pi \rho_{c} m_{c} \varphi_{1}\left(\sigma_{c}\right) \Theta\left(\sigma_{c}\right) \\
& \left.+\frac{1}{2 \Delta}\left(\sigma_{b} \frac{\varphi_{1}\left(\sigma_{a}\right)}{\varphi_{0}\left(\sigma_{a}\right)}+\sigma_{a} \frac{\varphi_{1}\left(\sigma_{b}\right)}{\varphi_{0}\left(\sigma_{b}\right)}\right) \sum_{c} \pi \rho_{c} m_{c} \varphi_{1}\left(\sigma_{c}\right) \sigma_{c} \psi\left(\sigma_{c}\right)\right] \\
& +e^{-s \sigma_{a}} \frac{\delta_{a b}}{2 \pi \rho_{a} m_{a}} \frac{K_{a}}{1-K_{a}} \frac{1}{\varphi_{0}\left(\sigma_{a}\right)}\left(\delta_{\alpha A} \delta_{\beta B}+\delta_{\alpha B} \delta_{\beta A}\right),
\end{aligned}
$$

where

$$
\begin{aligned}
& K_{a}=\left(\hat{\mathbf{g}}^{(a)} \hat{\mathbf{h}}^{(a)}\right)=\left(\hat{\mathbf{e}}^{(a)} \hat{\mathbf{f}}^{(a)}\right)=\frac{m_{a}-1}{2 m_{a} \sigma_{a}} \varphi_{0}\left(\sigma_{a}\right), \\
& \psi\left(\sigma_{a}\right)=1+\frac{2 K_{a}}{1-K_{a}}, \\
& \Theta\left(\sigma_{a}\right)=1+\frac{2 K_{a}}{1-K_{a}} \frac{\varphi_{1}\left(\sigma_{a}\right)}{\varphi_{0}\left(\sigma_{a}\right)} s,
\end{aligned}
$$

and

$$
\begin{aligned}
D & =1-\frac{2}{\Delta} \sum_{t} \pi \rho_{t} m_{t} \varphi_{2}\left(\sigma_{t}\right) \Theta\left(\sigma_{t}\right)\left\{1+\frac{1}{2 \Delta} \sum_{c} \pi \rho_{c} m_{c} \sigma_{c}^{3} \psi\left(\sigma_{c}\right)\right\} \\
& -\frac{1}{\Delta^{2}} \sum_{t} \pi \rho_{t} m_{t} \varphi_{2}\left(\sigma_{t}\right) \sigma_{t} \psi\left(\sigma_{t}\right)\left\{\xi_{2}-\sum_{c} \pi \rho_{c} m_{c} \sigma_{c}^{2} \Theta\left(\sigma_{c}\right)\right\} \\
& -\frac{2}{\Delta} \sum_{c} \pi \rho_{c} m_{c} \varphi_{1}\left(\sigma_{c}\right)\left\{\sigma_{c}\left(\psi\left(\sigma_{c}\right)\left[\frac{1}{2}+\frac{\xi_{2} \sigma_{c}}{4 \Delta}\right]+\frac{\Theta\left(\sigma_{c}\right)}{2}\right)\right. \\
& \left.+\frac{1}{4 \Delta} \psi\left(\sigma_{c}\right) \sum_{c} \pi \rho_{c} m_{c} \varphi_{1}\left(\sigma_{t}\right) \Theta\left(\sigma_{c}\right)\left(\sigma_{c}-\sigma_{t}\right)^{2}\right\} .
\end{aligned}
$$

Finally for the Laplace transform of the overall RDF we have

$$
G_{\mathrm{ref}}^{a b}(s)=\sum_{\alpha \beta} G_{\alpha \beta}^{a b}(s)
$$

The intermolecular and intramolecular parts of the overall RDFs are related to partial RDFs by

$$
\begin{aligned}
& G_{\text {inter }}^{a b}(s)=G_{00}^{a b}(s)+2 G_{A 0}^{a b}(s)+2 G_{0 A}^{a b}(s)+4 G_{A A}^{a b}(s), \\
& G_{\text {intra }}^{a b}(s)=2\left(G_{A B}^{a b}(s)-G_{A A}^{a b}(s)\right) .
\end{aligned}
$$




\section{THERMODYNAMIC PROPERTIES OF THE HARD-SPHERE YUKAWA CHAIN MIXTURE}

For the sake of simplicity we will consider here polydisperse mixture of the chains of different length and equal monomer size. Extension of the theory for the mixture with different chain monomer sizes is rather straightforward. According to TPT $[20,21]$ we have

$$
f_{\text {ref }}=f_{\text {id }}+f_{\mathrm{HS}}^{\mathrm{ex}}+f_{\text {chain }},
$$

where $f_{i d}$ is the ideal gas contribution

$$
\beta f_{\mathrm{id}}=\sum_{c} \rho_{c} m_{c}\left[\ln \left(\Lambda_{c}^{3} \rho_{c}\right)-1\right]
$$

with $\Lambda_{c}$ being thermal de Broglie wave length, $f_{\mathrm{HS}}^{\mathrm{ex}}$ is hard sphere excess free energy, which is represented here by the Carnahan-Starling expression [28]

$$
\beta f_{\mathrm{HS}}^{\mathrm{ex}}=\frac{1}{2 \pi \Delta} l^{2} \sigma^{3}\left(1+\frac{1}{3 \Delta}\right)
$$

and the third term in (61) contains contribution from the chain formation

$$
\beta f_{\text {chain }}=\sum_{c} \rho_{c}\left(m_{c}-1\right)\left[-\ln \left(4 \pi \rho_{c} \sigma_{c}^{2} Y\right)+1\right],
$$

where hard-sphere cavity correlation function $Y$ is calculated here using Carnahan-Starling approximation [28]

$$
Y=\frac{1}{\Delta}+\frac{l \sigma^{3}}{4 \Delta^{2}}+\frac{l^{2} \sigma^{6}}{72 \Delta^{3}}
$$

Finally, substituting expression for the RDF Laplace transform (58) into expression for the Yukawa contribution to Helmholtz free energy (4) and using (53), we get

$$
\begin{aligned}
\beta \Delta f_{\mathrm{HTA}} & =-\frac{2 \beta \epsilon_{0} r_{0}}{\pi \Delta D}\left\{l^{2}\left[\frac{1}{z_{0}^{2}}+\frac{\sigma}{z_{0}}-\frac{\sigma^{3}}{\Delta z_{0}} \frac{\varphi_{1}(\sigma)}{\varphi_{0}(\sigma)} v+\frac{l \sigma^{4}}{4 \Delta z_{0}}+\frac{\sigma^{3}}{2 \Delta z_{0}^{2}}(l+2 v)\right]\right. \\
& +4 l v\left[\frac{1}{z_{0}^{2}}+\frac{\sigma}{z_{0}}-\frac{\sigma}{\varphi_{0}(\sigma)}\left(\frac{1}{z_{0}^{2}}+\frac{\sigma}{2 z_{0}}\right)-\frac{\sigma^{3}}{\Delta z_{0}} \frac{\varphi_{1}(\sigma)}{\varphi_{0}(\sigma)} v+\frac{l \sigma^{4}}{4 \Delta z_{0}}+\frac{(l+2 v) \sigma^{3}}{2 \Delta z_{0}(\sigma)} \frac{\varphi_{0}(\sigma)}{\varphi_{0}(\sigma)}\right. \\
& +4 v^{2}\left[\left(\frac{\varphi_{1}(\sigma)}{\varphi_{0}(\sigma)}\right)^{2}+\sigma \frac{\varphi_{1}(\sigma)}{\varphi_{0}(\sigma)}+\frac{l \sigma^{4}}{4 \Delta z_{0}}+\frac{l+2 v}{\Delta}\left(\left(\frac{\sigma^{3}}{2}+\frac{\varphi_{1}(\sigma) z_{0} \sigma^{2}}{2}\right)\left(\frac{\varphi_{1}(\sigma)}{\varphi_{0}(\sigma)}\right)^{2}-\right.\right. \\
& \left.\left.\left.-\sigma^{2} \varphi_{1}(\sigma) \frac{\varphi_{1}(\sigma)}{\varphi_{0}(\sigma)}\right)+\frac{\left(l+2 v \frac{\varphi_{1}(\sigma)}{\varphi_{0}(\sigma)} z_{0}\right) \sigma^{2} \varphi_{1}(\sigma)}{2 \Delta z_{0}}\right]\right\}-\frac{2 \beta \epsilon_{0} r_{0}}{\pi} \frac{2 v}{\varphi_{0}(\sigma)}
\end{aligned}
$$

where

$$
\begin{gathered}
D=1-\frac{2}{\Delta} \varphi_{2}(\sigma)\left(l+2 v \frac{\varphi_{1}(\sigma)}{\varphi_{0}(\sigma)} s\right)\left(1+\frac{\sigma^{3}}{2 \Delta}(l+2 v)\right)+\frac{\sigma^{3} \varphi_{2}(\sigma)}{\Delta^{2}}(l+2 v) 2 v \frac{\varphi_{1}(\sigma)}{\varphi_{0}(\sigma)} s \\
-\frac{1}{\Delta} \varphi_{1}(\sigma) \sigma\left[(l+2 v)\left(1+\frac{l \sigma^{3}}{2 \Delta}\right)+\left(l+2 v \frac{\varphi_{1}(\sigma)}{\varphi_{0}(\sigma)} s\right)\right] \\
\varphi_{2}\left(\sigma_{b}\right)=\frac{1-z_{0} \sigma_{b}+z_{0}^{2} \sigma_{b}^{2} / 2-e^{-z_{0} \sigma_{b}}}{z_{0}^{3}}, \\
\varphi_{1}\left(\sigma_{b}\right)=\frac{1-z_{0} \sigma_{b}-e^{-z_{0} \sigma_{b}}}{z_{0}^{2}}, \quad \varphi_{0}\left(\sigma_{b}\right)=\frac{1-e^{-z_{0} \sigma_{b}}}{z_{0}} .
\end{gathered}
$$

In expressions (63), (65), (67) and (67)

$$
l=\pi \sum_{c} \rho_{c} m_{c}, \quad v=\pi \sum_{c} \rho_{c} m_{c} \frac{K_{c}}{1-K_{c}},
$$

are the moments of the chain species distribution function.

Expressions for the pressure of the system and chemical potential of the chain molecules can be obtained from the above expression for Helmholtz free energy (61)-(67). We have

$$
\begin{aligned}
& \beta P=\beta \sum_{c} \rho_{c} \mu_{c}-\beta f=\beta P_{\mathrm{HS}}+\beta P_{\text {chain }}+\beta \Delta P_{\mathrm{HTA}}, \\
& \beta \mu_{c}=\frac{\partial(\beta f)}{\partial \rho_{c}}=\beta \mu_{c, \mathrm{id}}+\beta \mu_{c, \mathrm{HS}}^{\mathrm{ex}}+\beta \mu_{c, \text { chain }}+\beta \Delta \mu_{c, \mathrm{HTA}} .
\end{aligned}
$$


Here $\mu_{c, \text { id }}, \mu_{c, \mathrm{HS}}^{\mathrm{ex}}, \mu_{c, \text { chain }}, \Delta \mu_{c, \mathrm{HTA}}$ and $P_{\text {chain }}, \Delta P_{\mathrm{HTA}}$ hold contributions from the corresponding free energies $f_{\text {id }}$, $f_{\mathrm{HS}}^{\mathrm{ex}}, f_{\text {chain }}$ and $\Delta f_{\mathrm{HTA}}$, except for $\beta P_{\mathrm{HS}}$ that holds a sum of contributions from $f_{\mathrm{id}}$ and $f_{\mathrm{HS}}^{\text {ex }}$. These expressions for excess chemical potential and pressure can be formulated in terms of the moments and their density derivatives

$$
\begin{aligned}
& \beta \mu_{c, \mathrm{id}}=m_{c} \ln \left\{\Lambda_{\mathrm{c}}^{3} \rho_{\mathrm{c}}\right\}, \\
& \beta \mu_{c, \mathrm{HS}}^{\mathrm{ex}}=\frac{l \sigma^{3} l(c)}{\pi \Delta}\left(1+\frac{1}{3 \Delta}\right)+\frac{l^{2} \sigma^{6} l(c)}{12 \pi \Delta^{2}}\left(1+\frac{2}{3 \Delta}\right), \\
& \beta \mu_{c, \text { chain }}=-\left(m_{c}-1\right) \ln \left\{4 \pi \rho_{\mathrm{c}} \sigma^{2} \mathrm{Y}\right\}-\frac{(1-\pi \rho)}{\mathrm{Y}} \frac{\partial \mathrm{Y}}{\partial \rho_{\mathrm{c}}}, \\
& \beta \Delta \mu_{c, \mathrm{HTA}}=-\frac{4 \beta \epsilon_{0} r_{0} e^{z_{0} \sigma}}{\pi} \pi \rho_{c} m_{c} \sum_{a} \pi \rho_{a} m_{a} G_{\mathrm{ref}}^{a c}\left(z_{0}\right)-\frac{2 \beta \epsilon_{0} r_{0} e^{z_{0} \sigma}}{\pi} \sum_{a} \pi \rho_{a} m_{a} \sum_{b} \pi \rho_{b} m_{b} \frac{\partial G_{\mathrm{ref}}^{a b}\left(z_{0}\right)}{\partial \rho_{c}}, \\
& \beta P_{\mathrm{HS}}=\frac{l}{\Delta \pi}\left(1+\frac{l \sigma^{3}}{2 \Delta}+\frac{l^{2} \sigma^{6}}{12 \Delta^{2}}+\frac{l^{3} \sigma^{9}}{216 \Delta^{2}}\right), \\
& \beta P_{\mathrm{chain}}=\frac{l-\pi \rho}{\pi}\left(\frac{1}{Y}\left[\frac{5 l \sigma^{3}}{12 \Delta^{2}}+\frac{l^{2} \sigma^{6}}{12 \Delta^{3}}\right]+1\right), \\
& \beta \Delta P_{\mathrm{HTA}}=-\frac{2 \beta \epsilon_{0} r_{0} e^{z_{0} \sigma}}{\pi} \sum_{a} \pi \rho_{a} m_{a} \sum_{b} \pi \rho_{b} m_{b} G_{\mathrm{ref}}^{a c}\left(z_{0}\right)-\frac{2 \beta \epsilon_{0} r_{0} e^{z_{0} \sigma}}{\pi} \sum_{a} \pi \rho_{a} m_{a} \sum_{b} \pi \rho_{b} m_{b} \sum_{c} \rho_{c} \frac{\partial G_{\mathrm{ref}}^{a b}\left(z_{0}\right)}{\partial \rho_{c}}
\end{aligned}
$$

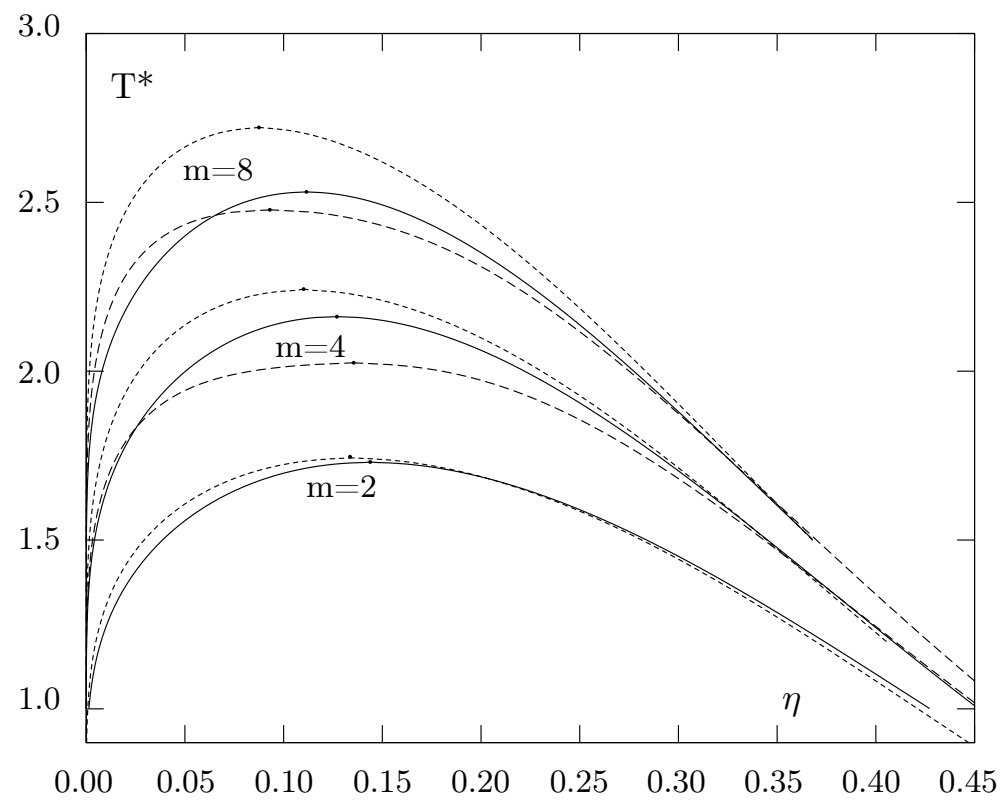

Fig. 1. Liquid-gas phase diagram for the systems with three different chain lengths $m=2,4,8$ from the bottom to the top. Solid curves represent HTA predictions, dotted curves represent SAFT-VR predictions, and dashed curves represent TPTD predictions. Small filled circle on the top of each coexistence curve shows the location of the corresponding critical point.

where

$$
\begin{aligned}
\frac{\partial Y}{\partial \rho_{c}} & =\frac{5 \sigma^{3} l_{c}}{12 \Delta^{3}}+\frac{l \sigma^{6} l_{c}}{9 \Delta^{2}}+\frac{l^{3} \sigma^{9} l_{c}}{144 \Delta^{4}} \\
l_{c} & =\frac{\partial l}{\partial \rho_{c}}=\pi m_{c}, \quad v_{c}=\frac{\partial v}{\partial \rho_{c}}=\pi m_{c} \frac{K_{c}}{1-K_{c}} .
\end{aligned}
$$

and $\rho=\sum_{c} \rho_{c}$ is total number density. Because of the size, final expressions for $\Delta \mu_{c, \mathrm{HTA}}$ and $\Delta P_{\mathrm{HTA}}$ via the moments are shown in the Appendix B.

We can conclude that our model belongs to the family of the TFE models since its thermodynamic properties can be represented by a finite number of (generalized) density distribution moments.

\section{RESULTS AND DISCUSSION}

To verify the accuracy of the present HTA approach we consider the limiting case of monodisperse one-Yukawa chain fluid and calculate liquid-gas phase diagram for several choices of the chain length. Unfortunately computer simulation results for the phase behavior of such 
systems are currently not available. Therefore we compare our results with those of the SAFT-VR approach and TPTD theory [19]. Recently the SAFT-VR and TPTD phase behavior predictions for two-Yukawa chain fluid were tested against computer simulation results [19]. According to this study the TPTD results appear to be more accurate of the two.

In Figure 1 we show liquid-gas phase diagram for Yukawa hard-sphere chain fluid with the chain length $m=2,4,8$ and Yukawa screening length $z_{0} r_{0}=1.8$. It can be seen that the HTA curve is located in between the corresponding TPTD and SAFT-VR curves. Note that in the previous study [19] the results of the TPTD approach were always slightly above computer simulation results. Here the HTA curve is located slightly above the TPTD curve. With the increase of the chain length the HTA and TPTD results become closer. Thus we can conclude that our HTA approach is more accurate than the SAFT-VR approach and slightly less accurate than TPTD.

\section{CONCLUDING REMARKS}

In this paper recently proposed HTA for polydisperse mixture of Yukawa hard spheres [7] is extended and applied to polydisperse mixture of Yukawa hard-sphere chain fluid. We consider the case of chain length polydispersity, although extension of the theory for the systems with both chain length and monomer size polydispersity is rather straightforward. We propose an averaged version of the multidensity $\mathrm{OZ}$ equation and use it to calculate the structure properties of the reference system, which are needed as an input in the HTA. The analytical solution of the OZ equation supplemented with the PPY approximation was used to derive a closed form analytical expression for Laplace transform of site-site RDFs of the system. Thermodynamical properties were calculated using TPT for associating fluids. In spite of its simplicity predictions of the HTA for the phase behavior of one-Yukawa chain fluid with monodispersity in the chain length appears to be more accurate than the corresponding predictions of SAFT-VR theory and slightly less accurate in comparison with the TPTD predictions.

According to our analysis polydisperse Yukawa hardsphere chain mixture treated within HTA belongs to the TFE class of models which allows one to study its phase behavior. The results of the corresponding studies will be presented in due course.

Acknowledgment This work was supported by the Science \& Technology Center in Ukraine through research grant No. 4140.

\section{APPENDIX A: COEFFICIENTS OF INVERSE MATRIX}

Presented below expressions for the inverse matrix coefficients take into account orthogonality conditions (48)-(51). Here the quantities in brackets $(\hat{\mathbf{x}} \hat{\mathbf{y}})$ represent a dot product of vectors $\hat{\mathbf{x}}$ and $\hat{\mathbf{y}}$. The first four coefficients are given by

$$
\begin{aligned}
\alpha_{\hat{a} \hat{b}} & =\frac{1-(\hat{\mathbf{d}} \hat{\mathbf{c}})-\sum_{t} \frac{\left(\hat{\mathbf{d}} \hat{\mathbf{e}}^{(t)}\right)\left(\hat{\mathbf{f}}^{(t)} \hat{\mathbf{c}}\right)}{1-K_{t}}-\sum_{t} \frac{\left(\hat{\mathbf{d}} \hat{\mathbf{g}}^{(t)}\right)\left(\hat{\mathbf{h}}^{(t)} \hat{\mathbf{c}}\right)}{1-K_{t}}}{D}, \\
\alpha_{\hat{c} \hat{d}} & =\frac{1-(\hat{\mathbf{b}} \hat{\mathbf{a}})-\sum_{t} \frac{\left(\hat{\mathbf{b}} \hat{\mathbf{e}}^{(t)}\right)\left(\hat{\mathbf{f}}^{(t)} \hat{\mathbf{a}}\right)}{1-K_{t}}-\sum_{t} \frac{\left(\hat{\mathbf{b}} \hat{\mathbf{g}}^{(t)}\right)\left(\hat{\mathbf{h}}^{(t)} \hat{\mathbf{a}}\right)}{1-K_{t}}}{D}, \\
\alpha_{\hat{a} \hat{d}} & =\frac{(\hat{\mathbf{b}} \hat{\mathbf{c}})+\sum_{t} \frac{\left(\hat{\mathbf{b}} \hat{\mathbf{e}}^{(t)}\right)\left(\hat{\mathbf{f}}^{(t)} \hat{\mathbf{c}}\right)}{1-K_{t}}+\sum_{t} \frac{\left(\hat{\mathbf{b}} \hat{\mathbf{g}}^{(t)}\right)\left(\hat{\mathbf{h}}^{(t)} \hat{\mathbf{c}}\right)}{1-K_{t}}}{D}, \\
\alpha_{\hat{c} \hat{b}}= & \frac{(\hat{\mathbf{d}} \hat{\mathbf{a}})+\sum_{t} \frac{\left(\hat{\mathbf{d}} \hat{\mathbf{e}}^{(t)}\right)\left(\hat{\mathbf{f}}^{(t)} \hat{\mathbf{a}}\right)}{1-K_{t}}+\sum_{t} \frac{\left(\hat{\mathbf{d}} \hat{\mathbf{g}}^{(t)}\right)\left(\hat{\mathbf{h}}^{(t)} \hat{\mathbf{a}}\right)}{1-K_{t}}}{D}
\end{aligned}
$$

where

$$
\begin{aligned}
D= & {\left[1-(\hat{\mathbf{d}} \hat{\mathbf{c}})-\sum_{t} \frac{\left(\hat{\mathbf{d}} \hat{\mathbf{e}}^{(t)}\right)\left(\hat{\mathbf{f}}^{(t)} \hat{\mathbf{c}}\right)}{1-K_{t}}-\sum_{t} \frac{\left(\hat{\mathbf{d}} \hat{\mathbf{g}}^{(t)}\right)\left(\hat{\mathbf{h}}^{(t)} \hat{\mathbf{c}}\right)}{1-K_{t}}\right][1-(\hat{\mathbf{b}} \hat{\mathbf{a}})} \\
& \left.-\sum_{t} \frac{\left(\hat{\mathbf{b}} \hat{\mathbf{e}}^{(t)}\right)\left(\hat{\mathbf{f}}^{(t)} \hat{\mathbf{a}}\right)}{1-K_{t}}-\sum_{t} \frac{\left(\hat{\mathbf{b}} \hat{\mathbf{g}}^{(t)}\right)\left(\hat{\mathbf{h}}^{(t)} \hat{\mathbf{a}}\right)}{1-K_{t}}\right]-\left[(\hat{\mathbf{b}} \hat{\mathbf{c}})+\sum_{t} \frac{\left(\hat{\mathbf{b}} \hat{\mathbf{e}}^{(t)}\right)\left(\hat{\mathbf{f}}^{(t)} \hat{\mathbf{c}}\right)}{1-K_{t}}\right.
\end{aligned}
$$




$$
\left.+\sum_{t} \frac{\left(\hat{\mathbf{b}} \hat{\mathbf{g}}^{(t)}\right)\left(\hat{\mathbf{h}}^{(t)} \hat{\mathbf{c}}\right)}{1-K_{t}}\right]\left[(\hat{\mathbf{d}} \hat{\mathbf{a}})+\sum_{t} \frac{\left(\hat{\mathbf{d}} \hat{\mathbf{e}}^{(t)}\right)\left(\hat{\mathbf{f}}^{(t)} \hat{\mathbf{a}}\right)}{1-K_{t}}+\sum_{t} \frac{\left(\hat{\mathbf{d}} \hat{\mathbf{g}}^{(t)}\right)\left(\hat{\mathbf{h}}^{(t)} \hat{\mathbf{a}}\right)}{1-K_{t}}\right],
$$

and $K_{t}=\left(\hat{\mathbf{f}}^{(t)} \hat{\mathbf{e}}^{(t)}\right)=\left(\hat{\mathbf{g}}^{(t)} \hat{\mathbf{h}}^{(t)}\right)$.

The remaining twelve coefficients are as follows:

$$
\begin{aligned}
& \alpha_{\hat{e} \hat{b}}^{(p)}=\frac{1}{1-K_{p}}\left\{\alpha_{\hat{a} \hat{b}}\left(\hat{\mathbf{f}}^{(p)} \hat{\mathbf{a}}\right)+\alpha_{\hat{c} \hat{b}}\left(\hat{\mathbf{f}}^{(p)} \hat{\mathbf{c}}\right)\right\}, \\
& \alpha_{\hat{g} \hat{b}}^{(p)}=\frac{1}{1-K_{p}}\left\{\alpha_{\hat{a} \hat{b}}\left(\hat{\mathbf{h}}^{(p)} \hat{\mathbf{a}}\right)+\alpha_{\hat{c} \hat{b}}\left(\hat{\mathbf{h}}^{(p)} \hat{\mathbf{c}}\right)\right\}, \\
& \alpha_{\hat{e} \hat{d}}^{(p)}=\frac{1}{1-K_{p}}\left\{\alpha_{\hat{a} \hat{d}}\left(\hat{\mathbf{f}}^{(p)} \hat{\mathbf{a}}\right)+\alpha_{\hat{c} \hat{d}}\left(\hat{\mathbf{f}}^{(p)} \hat{\mathbf{c}}\right)\right\}, \\
& \alpha_{\hat{g} \hat{d}}^{(p)}=\frac{1}{1-K_{p}}\left\{\alpha_{\hat{a} \hat{d}}\left(\hat{\mathbf{h}}^{(p)} \hat{\mathbf{a}}\right)+\alpha_{\hat{c} \hat{d}}\left(\hat{\mathbf{h}}^{(p)} \hat{\mathbf{c}}\right)\right\}, \\
& \alpha_{\hat{a} \hat{f}}^{(p)}=\frac{1}{1-K_{p}}\left\{\alpha_{\hat{a} \hat{b}}\left(\hat{\mathbf{b}} \hat{\mathbf{e}}^{(p)}\right)+\alpha_{\hat{a} \hat{d}}\left(\hat{\mathbf{d}} \hat{\mathbf{e}}^{(p)}\right)\right\}, \\
& \alpha_{\hat{c} \hat{f}}^{(p)}=\frac{1}{1-K_{p}}\left\{\alpha_{\hat{c} \hat{b}}\left(\hat{\mathbf{b}} \hat{\mathbf{e}}^{(p)}\right)+\alpha_{\hat{c} \hat{d}}\left(\hat{\mathbf{d}} \hat{\mathbf{e}}^{(p)}\right)\right\}, \\
& \alpha_{\hat{a} \hat{h}}^{(p)}=\frac{1}{1-K_{p}}\left\{\alpha_{\hat{a} \hat{b}}\left(\hat{\mathbf{b}} \hat{\mathbf{g}}^{(p)}\right)+\alpha_{\hat{a} \hat{d}}\left(\hat{\mathbf{d}} \hat{\mathbf{g}}^{(p)}\right)\right\}, \\
& \alpha_{\hat{c} \hat{h}}^{(p)}=\frac{1}{1-K_{p}}\left\{\alpha_{\hat{c} \hat{b}}\left(\hat{\mathbf{b}}_{\hat{\mathbf{g}}}^{(p)}\right)+\alpha_{\hat{c} \hat{d}}\left(\hat{\mathbf{d}} \hat{\mathbf{g}}^{(p)}\right)\right\}, \\
& \alpha_{\hat{e} \hat{f}}^{(p q)} \frac{\delta^{(p q)}}{1-K_{p}}+\frac{\alpha_{\hat{a} \hat{d}}\left(\hat{\mathbf{d}}^{(q)}\right)\left(\hat{\mathbf{f}}^{(p)} \hat{\mathbf{a}}\right)+\alpha_{\hat{a} \hat{b}}\left(\hat{\mathbf{b}} \hat{\mathbf{e}}^{(q)}\right)\left(\hat{\mathbf{f}}^{(p)} \hat{\mathbf{a}}\right)+\alpha_{\hat{c} \hat{d}}\left(\hat{\mathbf{d}} \hat{\mathbf{e}}^{(q)}\right)\left(\hat{\mathbf{f}}^{(p)} \hat{\mathbf{c}}\right)+\alpha_{\hat{c} \hat{b}}\left(\hat{\mathbf{b}} \hat{\mathbf{e}}^{(q)}\right)\left(\hat{\mathbf{f}}^{(p)} \hat{\mathbf{c}}\right)}{\left(1-K_{p}\right)\left(1-K_{q}\right)} \\
& \alpha_{\hat{e} \hat{h}}^{(p q)}=\frac{\alpha_{\hat{a} \hat{d}}\left(\hat{\mathbf{d}} \hat{\mathbf{g}}^{(q)}\right)\left(\hat{\mathbf{f}}^{(p)} \hat{\mathbf{a}}\right)+\alpha_{\hat{a} \hat{b}}\left(\hat{\mathbf{b}} \hat{\mathbf{g}}^{(q)}\right)\left(\hat{\mathbf{f}}^{(p)} \hat{\mathbf{a}}\right)+\alpha_{\hat{c} \hat{d}}\left(\hat{\mathbf{d}} \hat{\mathbf{g}}^{(q)}\right)\left(\hat{\mathbf{f}}^{(p)} \hat{\mathbf{c}}\right)+\alpha_{\hat{c} \hat{b}}\left(\hat{\mathbf{b}} \hat{\mathbf{g}}^{(q)}\right)\left(\hat{\mathbf{f}}^{(p)} \hat{\mathbf{c}}\right)}{\left(1-K_{p}\right)\left(1-K_{q}\right)}, \\
& \alpha_{\hat{g} \hat{h}}^{(p q)} \frac{\delta^{(p q)}}{1-K_{p}}+\frac{\alpha_{\hat{a} \hat{d}}\left(\hat{\mathbf{d}} \hat{\mathbf{g}}^{(q)}\right)\left(\hat{\mathbf{h}}^{(p)} \hat{\mathbf{a}}\right)+\alpha_{\hat{a} \hat{b}}\left(\hat{\mathbf{b}} \hat{\mathbf{g}}^{(q)}\right)\left(\hat{\mathbf{h}}^{(p)} \hat{\mathbf{a}}\right)+\alpha_{\hat{c} \hat{d}}\left(\hat{\mathbf{d}} \hat{\mathbf{g}}^{(q)}\right)\left(\hat{\mathbf{h}}^{(p)} \hat{\mathbf{c}}\right)+\alpha_{\hat{c} \hat{b}}\left(\hat{\mathbf{b}} \hat{\mathbf{g}}^{(q)}\right)\left(\hat{\mathbf{h}}^{(p)} \hat{\mathbf{c}}\right)}{\left(1-K_{p}\right)\left(1-K_{q}\right)}, \\
& \alpha_{\hat{g} \hat{f}}^{(p)}=\frac{\alpha_{\hat{a} \hat{d}}\left(\hat{\mathbf{d}} \hat{\mathbf{e}}^{(q)}\right)\left(\hat{\mathbf{h}}^{(p)} \hat{\mathbf{a}}\right)+\alpha_{\hat{a} \hat{b}}\left(\hat{\mathbf{b}} \hat{\mathbf{e}}^{(q)}\right)\left(\hat{\mathbf{h}}^{(p)} \hat{\mathbf{a}}\right)+\alpha_{\hat{c} \hat{d}}\left(\hat{\mathbf{d}} \hat{\mathbf{e}}^{(q)}\right)\left(\hat{\mathbf{h}}^{(p)} \hat{\mathbf{c}}\right)+\alpha_{\hat{c} \hat{b}}\left(\hat{\mathbf{b}} \hat{\mathbf{e}}^{(q)}\right)\left(\hat{\mathbf{h}}^{(p)} \hat{\mathbf{c}}\right)}{\left(1-K_{p}\right)\left(1-K_{q}\right)} .
\end{aligned}
$$

\section{APPENDIX B: EXPRESSIONS FOR THE CHEMICAL POTENTIAL AND PRESSURE OF THE REFERENCE SYSTEM}

The quantities given below can be simplified, but they are kept here in the form that resembles the structure of the overall RDFs Laplace transform.

The HTA contribution to the chemical potential reads

$$
\begin{aligned}
& \beta \Delta \mu_{c, H T A}=-\frac{4 \beta \epsilon_{0} r_{0}}{\pi \Delta D}\left\{l l_{c}\left[\frac{1}{z_{0}^{2}}+\frac{\sigma}{z_{0}}-\frac{\sigma^{3}}{\Delta z_{0}} \frac{\varphi_{1}(\sigma)}{\varphi_{0}(\sigma)} v+\frac{l \sigma^{4}}{4 \Delta z_{0}}+\frac{\sigma^{3}}{2 \Delta z_{0}^{2}}(l+2 v)\right]\right. \\
& +2\left(v l_{c}+l v_{c}\right)\left[\frac{1}{z_{0}^{2}}+\frac{\sigma}{z_{0}}-\frac{\sigma}{\varphi_{0}(\sigma)}\left(\frac{1}{z_{0}^{2}}+\frac{\sigma}{2 z_{0}}\right)-\frac{\sigma^{3}}{\Delta z_{0}} \frac{\varphi_{1}(\sigma)}{\varphi_{0}(\sigma)} v+\frac{l \sigma^{4}}{4 \Delta z_{0}}\right. \\
& \left.+\frac{(l+2 v) \sigma^{3}}{2 \Delta z_{0}} \frac{\varphi_{1}(\sigma)}{\varphi_{0}(\sigma)}\right]+4 v v_{c}\left[\left(\frac{\varphi_{1}(\sigma)}{\varphi_{0}(\sigma)}\right)^{2}+\sigma \frac{\varphi_{1}(\sigma)}{\varphi_{0}(\sigma)}+\frac{l \sigma^{4}}{4 \Delta z_{0}}\right. \\
& \left.\left.+\frac{l+2 v}{\Delta}\left(\left(\frac{\sigma^{3}}{2}+\frac{\varphi_{1}(\sigma) z_{0} \sigma^{2}}{2}\right)\left(\frac{\varphi_{1}(\sigma)}{\varphi_{0}(\sigma)}\right)^{2}-\sigma^{2} \varphi_{1}(\sigma) \frac{\varphi_{1}(\sigma)}{\varphi_{0}(\sigma)}\right)+\frac{\left(l 2 v \frac{\varphi_{1}(\sigma)}{\varphi_{0}(\sigma)} z_{0}\right) \sigma^{2} \varphi_{1}(\sigma)}{2 \Delta z_{0}}\right]\right\}
\end{aligned}
$$




$$
\begin{aligned}
& -\frac{4 \beta \epsilon_{0} r_{0}}{\pi} \frac{v_{c}}{\varphi_{0}(\sigma)}+\beta\left(f_{\mathrm{HTA}}-f_{\mathrm{HTA}}^{\text {intra. }}\right)\left(\frac{l_{c} \sigma^{3}}{6 \Delta}-\frac{\frac{\partial D}{\partial \rho_{c}}}{D}\right) \\
& -\frac{2 \beta \epsilon_{0} r_{0}}{\pi \Delta D}\left\{l l \left[-\frac{v_{c} \sigma^{3}}{\Delta z_{0}} \frac{\varphi_{1}(\sigma)}{\varphi_{0}(\sigma)}-\frac{v \sigma^{6} l_{c}}{6 \Delta^{2} z_{0}} \frac{\varphi_{1}(\sigma)}{\varphi_{0}(\sigma)}+\frac{l_{c} \sigma^{4}}{4 \Delta z_{0}}+\frac{l \sigma^{7} l_{c}}{24 \Delta^{2} z_{0}}+\frac{\sigma^{3}\left(l_{c}+2 v_{c}\right)}{2 \Delta z_{0}^{2}}\right.\right. \\
& \left.+\frac{(l 2 v) \sigma^{6} l_{c}}{12 \Delta^{2} z_{0}^{2}}\right]+4 l v\left[-\frac{\sigma^{3} v_{c}}{\Delta z_{0}} \frac{\varphi_{1}(\sigma)}{\varphi_{0}(\sigma)}-\frac{v \sigma^{6} l_{c}}{6 \Delta^{2} z_{0}} \frac{\varphi_{1}(\sigma)}{\varphi_{0}(\sigma)}+\frac{l_{c} \sigma^{4}}{4 \Delta z_{0}}+\frac{l \sigma^{7} l_{c}}{24 \Delta^{2} z_{0}}\right. \\
& \left.+\left(\frac{\sigma^{3}\left(l_{c}+2 v_{c}\right)}{2 \Delta z_{0}}+\frac{(l+2 v) \sigma^{6} l_{c}}{12 \Delta^{2} z_{0}}\right) \frac{\varphi_{1}(\sigma)}{\varphi_{0}(\sigma)}\right]+4 v v\left[\frac{l_{c} \sigma^{4}}{4 \Delta z_{0}}+\frac{l \sigma^{7} l_{c}}{24 \Delta^{2} z_{0}}\right. \\
& +\left(\frac{l_{c}+2 v_{c}}{\Delta}+\frac{(l+2 v) \sigma^{3} l_{c}}{6 \Delta^{2}}\right)\left(\left(\frac{\sigma^{3}}{2}+\frac{\varphi_{1}(\sigma) z_{0} \sigma^{2}}{2}\right)\left(\frac{\varphi_{1}(\sigma)}{\varphi_{0}(\sigma)}\right)^{2}-\sigma^{2} \varphi_{1}(\sigma) \frac{\varphi_{1}(\sigma)}{\varphi_{0}(\sigma)}\right) \\
& \left.\left.+\frac{\left(l_{c}+2 v_{c} \frac{\varphi_{1}(\sigma)}{\varphi_{0}(\sigma)} z_{0}\right) \sigma^{2} \varphi_{1}(\sigma)}{2 \Delta z_{0}}+\frac{\left(l+2 v \frac{\varphi_{1}(\sigma)}{\varphi_{0}(\sigma)} z_{0}\right) \sigma^{5} \varphi_{1}(\sigma) l_{c}}{12 \Delta^{2} z_{0}}\right]\right\}+\frac{2 \beta \epsilon_{0} r_{0}}{\pi} \frac{v_{c}}{\varphi_{0}(\sigma)}
\end{aligned}
$$

where

$$
\beta f_{\mathrm{HTA}}^{\mathrm{intra}}=-\frac{2 \beta \epsilon_{0} r_{0}}{\pi} \frac{2 v}{\varphi_{0}(\sigma)}
$$

and $\partial D / \partial \rho_{c}$ has the following form

$$
\begin{aligned}
\frac{\partial D}{\partial \rho_{c}}= & -\frac{\varphi_{2}(\sigma) \sigma^{3} l_{c}}{3 \Delta^{2}}\left(l+2 v \frac{\varphi_{1}(\sigma)}{\varphi_{0}(\sigma)} z_{0}\right)\left(1+\frac{\sigma^{3}}{2 \Delta}(l+2 v)\right) \\
& -\frac{2}{\Delta} \varphi_{2}(\sigma)\left[\left(l_{c}+2 v_{c} \frac{\varphi_{1}(\sigma)}{\varphi_{0}(\sigma)} z_{0}\right)\left(1+\frac{\sigma^{3}}{2 \Delta}(l+2 v)\right)\right. \\
& \left.+\left(l+2 v \frac{\varphi_{1}(\sigma)}{\varphi_{0}(\sigma)} z_{0}\right)\left(\frac{\sigma^{6} l_{c}}{12 \Delta^{2}}(l+2 v)+\frac{\sigma^{3}}{2 \Delta}\left(l_{c}+2 v_{c}\right)\right)\right]+\frac{\varphi_{2}(\sigma) \sigma^{6} l_{c}}{3 \Delta^{3}}(l+2 v) 2 v \frac{\varphi_{1}(\sigma)}{\varphi_{0}(\sigma)} z_{0} \\
& +\frac{\sigma^{3} \varphi_{2}(\sigma)}{\Delta^{2}}\left[\left(l_{c}+2 v_{c}\right) 2 v+(l+2 v) 2 v_{c}\right] \frac{\varphi_{1}(\sigma)}{\varphi_{0}(\sigma)} z_{0} \\
& -\frac{\sigma^{3} l_{c}}{3 \Delta^{2}} \varphi_{1}(\sigma) \sigma\left[(l+2 v)\left(1+\frac{l \sigma^{3}}{2 \Delta}\right)+\left(l+2 v \frac{\varphi_{1}(\sigma)}{\varphi_{0}(\sigma)} z_{0}\right)\right] \\
& -\frac{1}{\Delta} \varphi_{1}(\sigma) \sigma\left[\left(l_{c}+2 v_{c}\right)\left(1+\frac{l \sigma^{3}}{2 \Delta}\right)+(l+2 v)\left(\frac{\sigma^{3} l_{c}}{2 \Delta}+\frac{l \sigma^{6} l_{c}}{12 \Delta^{2}}\right)+\left(l_{c}+2 v_{c} \frac{\varphi_{1}(\sigma)}{\varphi_{0}(\sigma)} z_{0}\right)\right] .
\end{aligned}
$$

The corresponding expression for pressure is given by

$$
\begin{aligned}
& \Delta \beta P_{\mathrm{HTA}}=\beta f_{\mathrm{HTA}}+\beta\left(f_{\mathrm{HTA}}-f_{\mathrm{HTA}}^{\text {intra. }}\right)\left(\frac{l \sigma^{3}}{6 \Delta}-\frac{\sum_{c} \rho_{c} \frac{\partial D}{\partial \rho_{c}}}{D}\right) \\
& -\frac{2 \beta \epsilon_{0} r_{0}}{\pi \Delta D}\left\{l l \left[-\frac{v \sigma^{3}}{\Delta z_{0}} \frac{\varphi_{1}(\sigma)}{\varphi_{0}(\sigma)}-\frac{v \sigma^{6} l}{6 \Delta^{2} z_{0}} \frac{\varphi_{1}(\sigma)}{\varphi_{0}(\sigma)}+\frac{l \sigma^{4}}{4 \Delta z_{0}}+\frac{l \sigma^{7} l}{24 \Delta^{2} z_{0}}+\frac{\sigma^{3}(l+2 v)}{2 \Delta z_{0}^{2}}\right.\right. \\
& \left.+\frac{(l+2 v) \sigma^{6} l}{12 \Delta^{2} z_{0}^{2}}\right]+4 l v\left[-\frac{\sigma^{3} v}{\Delta z_{0}} \frac{\varphi_{1}(\sigma)}{\varphi_{0}(\sigma)}-\frac{v \sigma^{6} l}{6 \Delta^{2} z_{0}} \frac{\varphi_{1}(\sigma)}{\varphi_{0}(\sigma)}+\frac{l \sigma^{4}}{4 \Delta z_{0}}+\frac{l \sigma^{7} l}{24 \Delta^{2} z_{0}}\right. \\
& \left.+\left(\frac{\sigma^{3}(l+2 v)}{2 \Delta z_{0}}+\frac{(l+2 v) \sigma^{6} l}{12 \Delta^{2} z_{0}}\right) \frac{\varphi_{1}(\sigma)}{\varphi_{0}(\sigma)}\right]+4 v v\left[\frac{l \sigma^{4}}{4 \Delta z_{0}}+\frac{l \sigma^{7} l}{24 \Delta^{2} z_{0}}\right. \\
& +\left(\frac{l+2 v}{\Delta}+\frac{(l+2 v) \sigma^{3} l}{6 \Delta^{2}}\right)\left(\left(\frac{\sigma^{3}}{2}+\frac{\varphi_{1}(\sigma) z_{0} \sigma^{2}}{2}\right)\left(\frac{\varphi_{1}(\sigma)}{\varphi_{0}(\sigma)}\right)^{2}-\sigma^{2} \varphi_{1}(\sigma) \frac{\varphi_{1}(\sigma)}{\varphi_{0}(\sigma)}\right) \\
& \left.+\frac{\left(l+2 v \frac{\varphi_{1}(\sigma)}{\varphi_{0}(\sigma)} z_{0}\right) \sigma^{2} \varphi_{1}(\sigma)}{2 \Delta z_{0}}+\frac{\left.\left(l+2 v \frac{\varphi_{1}(\sigma)}{\varphi_{0}(\sigma)} z_{0}\right) \sigma^{5} \varphi_{1}(\sigma) l\right]}{12 \Delta^{2} z_{0}}\right]+\frac{2 \beta \epsilon_{0} r_{0}}{\pi} \frac{v}{\varphi_{0}(\sigma)},
\end{aligned}
$$

where $\sum_{c} \rho_{c} \frac{\partial D}{\partial \rho_{c}}$ can be obtained from (3) replacing $l_{c}$ and $v_{c}$ by $l=\sum_{c} \rho_{c} l_{c}$ and $v=\sum_{c} \rho_{c} v_{c}$. 
[1] J. J. Salacuse, G. Stell, J. Chem. Phys. 77, 3714 (1982).

[2] P. Sollich, J. Phys. 14, R79 (2002).

[3] L. Bellier-Castella, H. Xu, M. Baus, J. Chem. Phys. 113, 8337 (2000); 115, 3381 (2001).

[4] H. Xu, L. Bellier-Castello, M. Baus, J. Phys. 14, 12141 (2002).

[5] N. B. Wilding, M. Fasolo, P. Sollich, J. Chem. Phys. 121, 6887 (2004).

[6] Yu. V. Kalyuzhnyi, G. Kahl, J. Chem. Phys. 119, 7335 (2003).

[7] Yu. V. Kalyuzhnyi, S. P. Hlushak, J. Chem. Phys. 125, 034501 (2006).

[8] Yu. V. Kalyuzhnyi, G. Kahl, P. T. Cummings, J. Chem. Phys. 120, 10133 (2004).

[9] Yu. V. Kalyuzhnyi, G. Kahl, P. T. Cummings, Europhys. Lett. 72, 96 (2005).

[10] Yu. V. Kalyuzhnyi, G. Kahl, P. T. Cummings, J. Chem. Phys. 123, 124501 (2005).

[11] K. G. Honnell, C. K. Hall, J. Chem. Phys. 90, 1841 (1989); 95, 4481 (1991).

[12] K. S. Schweizer, J. G. Curro, Adv. Polymer Sci. 116, 320 (1994).

[13] A. Gil-Villegas, A. Galindo, P. J. Whitehead, S. J. Mills, G. Jackson, J. Chem. Phys. 106, 4168 (1997).
[14] L. A. Davies, A. Gil-Villegas, G. Jackson, J. Chem. Phys. 111, 8659 (1999).

[15] Yu. V. Kalyuzhnyi, C.-T. Lin, G. Stell, J. Chem. Phys. 106, 1940 (1997); 108, 6513, 6525 (1998).

[16] G. Stell, C.-T. Lin, Yu. V. Kalyuzhnyi, J. Chem. Phys. 110, 5444 (1999).

[17] Y. V. Kalyuzhnyi, Mol. Phys. 94, 735 (1998).

[18] Y. V. Kalyuzhnyi, C. McCabe, P. T. Cumings, G. Stell, Mol. Phys. 100, 2499 (2002).

[19] E. Whitebay, P. T. Cummings, Yu. V. Kalyuzhnyi, C. McCabe, J. Chem. Phys. 121, 8128 (2004).

[20] M. S. Wertheim, J. Stat. Phys. 42, 459 (1986).

[21] W. G. Chapman, G. Jackson, K. E. Gubbins, Mol. Phys. 65, 1057 (1988).

[22] J. Chang, S. I. Sandler, J. Chem. Phys. 102, 437 (1995).

[23] J. Chang, S. I. Sandler, J. Chem. Phys. 103, 3196 (1995).

[24] R. J. Baxter, J. Chem. Phys. 52, 4559 (1970).

[25] L. Blum, J. S. Hoye, J. Chem. Phys. 81, 1311 (1977).

[26] M. F. Golovko, I. A. Protsykevich, Chem. Phys. Lett. 142, 463 (1987)

[27] M. F. Holovko, I. A. Protsykevich, Condens. Matter Phys. 10, 137 (1997).

[28] N. F. Carnahan, K. E. Starling, J. Chem. Phys. 51, 635 (1969).

\title{
ПОЛІДИСПЕРСНА РІДИНА ЛАНЦЮГІВ ТВЕРДИХ СФЕР ІЗ ЮКАВІВСЬКОЮ ВЗАЄМОДІЄЮ: ВИСОКОТЕМПЕРАТУРНЕ НАБЛИЖЕННЯ
}

\author{
С. П. Глушак, Ю. В. Калюжний \\ Інститут фізики конденсованих систем НАН Украӥни \\ вул. Свєниічького, 1, Лъвів, 79011, Украӥна
}

\begin{abstract}
Для опису полідисперсної за довжиною рідини ланцюгів твердих сфер із Юкавіською взаємодією запропоновано високотемпературне наближення. Для застосування високотемпературного наближення з розв'язку усередненого багатогустинного рівняння Орштайна-Церніке отримано аналітичний вираз для Лапласобразу радіяльної функції розподілу між мономерами ланцюга. Також продемонстровано, що в межах високотемпературного наближення досліджувана система належить до класу моделей з "обрізаною" вільною енергією, тобто моделей, термодинамічні властивості яких (вільна енергія, хемічний потенціял та тиск) виражаються скінченною кількістю узагальнених моментів функції розподілу. Для порівняння точности отриманих результатів у роботі подано фазові діяграми співіснування монодисперсних газу та рідини для статистичної теорії асоціятивних рідин (SAFT-VR) та термодинамічної теорії збурень для димерів (TPTD).
\end{abstract}

